\title{
Anti-mouse RANKL Antibodies Inhibit Alveolar Bone Destruction in Periodontitis Model Mice
}

\author{
Miku Kuritani ${ }^{a, b, \#}$ Nobuhiro Sakai, ${ }^{a, \#}$ Akiko Karakawa, ${ }^{a}$ Motoki Isawa, ${ }^{a, c}$ Masahiro Chatani, ${ }^{a}$ \\ Takako Negishi-Koga,${ }^{a}$ Takahiro Funatsu, ${ }^{b}$ and Masamichi Takami*,a \\ ${ }^{a}$ Department of Pharmacology, School of Dentistry, Showa University; 1-5-8 Hatanodai, Shinagawa-ku, Tokyo, \\ 142-8555, Japan: ${ }^{b}$ Department of Special Needs Dentistry, Division of Dentistry for Persons with Disabilities, School \\ of Dentistry, Showa University; 2-1-1 Kitasenzoku, Ota-ku, Tokyo 145-8515, Japan: and ${ }^{c}$ Department of Pediatric \\ Dentistry, School of Dentistry, Showa University; 2-1-1 Kitasenzoku, Ota-ku, Tokyo 145-8515, Japan.
}

Received January 16, 2018; accepted January 24, 2018

\begin{abstract}
Denosumab is an anti-bone resorptive drug consisting of complete human monoclonal antibodies that targets receptor activator of nuclear factor $\kappa \mathrm{B}$ ligand (RANKL), which is responsible for osteoclast formation. The drug has been adapted for bone diseases, such as osteoporosis and bone metastasis related to cancer, but is not used for alveolar bone destruction related to periodontitis. In the present study, we aimed to clarify whether denosumab prevents bone destruction associated with lipopolysaccharide (LPS)-induced calvaria inflammation and experimental periodontitis in model mice. Denosumab does not bind to mouse RANKL, thus we used anti-mouse monoclonal RANKL antibodies. We also examined the inhibitory effects toward bone destruction of another anti-bone resorptive drug zoledronate, a nitrogen-containing bisphosphonate. Local administration of anti- RANKL antibodies into the calvaria area inhibited LPS-induced osteoclast formation and bone destruction, while zoledronate inhibited bone destruction but not osteoclast formation due to its different action mechanism. In periodontitis model mice, in which the second molars were ligated with a silk suture to induce inflammation, intraperitoneal administration of anti-RANKL antibodies significantly inhibited alveolar bone destruction and tooth root exposure. On the other hand, zoledronate only weakly repressed alveolar bone destruction and failed to inhibit root exposure. These results suggest that denosumab is a promising candidate to prevent alveolar bone destruction associated with periodontitis.
\end{abstract}

Key words denosumab; periodontitis; bone; osteoclast; molecularly targeted drug; bisphosphonate

Denosumab is a powerful anti-bone resorptive drug produced for treatment of osteoporosis patients who have a high risk for fracture, as well as development of cancer metastasis and giant cell tumors in bone tissue. ${ }^{1)}$ The drug consists of human monoclonal antibodies that bind to receptor activator of nuclear factor $\kappa \mathrm{B}$ ligand (RANKL), a tumor necrosis factor (TNF)-super family cytokine produced by osteoblasts and stromal cells in bone tissues. ${ }^{2)}$ RANKL plays roles in signal transduction for osteoclast differentiation and function by binding to its receptor RANK (receptor activator of nuclear factor $\kappa \mathrm{B}$ ) expressed on mature osteoclasts and their precursors. ${ }^{3-5)}$ Thus, denosumab inhibits bone destruction caused by enhanced differentiation and function of osteoclasts by interrupting RANKL and RANK interactions.

Destruction of alveolar bone occurs in patients with severe periodontitis, which is characterized by chronic gingival inflammation. ${ }^{6)}$ Dental plaque, which contains bacterial constituents such as lipopolysaccharide (LPS), stimulates osteoclast differentiation via production of inflammatory factors including interleukin (IL)-1, TNF- $\alpha$, and prostaglandins (PGs). ${ }^{7)}$ These factors enhance differentiation of osteoclasts on alveolar bone in both direct and indirect manners, resulting in tooth loss due to alveolar bone destruction. ${ }^{6)}$

Regular brushing is known to be the most effective means of keeping the periodontal area clean and preventing periodontitis. $^{8)}$ However, handicapped or bedridden individuals find it difficult to use a dental brush by themselves, and tend

\footnotetext{
${ }^{\#}$ These authors contributed equally to this work.

to have severe periodontitis, leading to tooth loss. Therefore, establishment of alternative methods for prevention of alveolar bone destruction accompanied by periodontitis is very important. One possible method is local administration of an anti-bone resorptive drug such as denosumab in alveolar bone areas. However, it is not known if that drug is effective for treatment and prevention of alveolar bone destruction caused by periodontitis.

In the present study, to clarify whether drug therapy using denosumab can prevent bone destruction in association with periodontitis, we examined the effects of anti-mouse RANKL monoclonal antibodies, used to mimic denosumab, on LPSinduced calvarial bone destruction and alveolar bone destruction related to periodontitis induced experimentally in mice. In addition, we examined the effects of the bisphosphonate zoledronate, another type of anti-bone resorptive drug, and compared them with those of the anti-mouse RANKL monoclonal antibodies in mouse models of bone destruction.

Our results showed that administration of anti-mouse RANKL monoclonal antibodies strongly inhibited calvarial and alveolar bone destruction, suggesting that denosumab may be a good candidate drug for treatment of periodontitisinduced alveolar bone destruction.

\section{MATERIALS AND METHODS}

Reagents LPS (Escherichia coli 026:B6, \#L2654) was purchased from Sigma-Aldrich Co., Ltd. (St. Louis, MO, U.S.A.), anti-mouse monoclonal RANKL antibodies $\left(\mathrm{OYC1}^{\circledR}\right)$ 
from ORIENTAL YEAST Co., Ltd. (Tokyo, Japan), zoledronate $\left(\right.$ ZOMETA $\left.^{\circledR}\right)$ from Novartis Pharma K.K. (Basel, Switzerland), physiological saline from Otsuka Pharmaceutical Co., Ltd. (Tokyo, Japan), and type I collagen gel (Cellmatrix type I A) from Nitta Gelatin Inc. (Osaka, Japan).

Animals and Ethical Approval Eight-week-old C57BL/6j male mice were purchased from Sankyo Labo Service Corporation, Inc. (Tokyo, Japan). All animal experiments were approved by the Showa University Animal Care and Use Committee (approval number: 17041), and conducted according to the ethical guidelines of that institution.

LPS-Induced Calvarial Bone Destruction Model Mice Mice were anesthetized with isoflurane inhalation, then collagen gel $(200 \mu \mathrm{L})$ containing LPS $(25 \mathrm{mg} / \mathrm{kg})$ with or without anti-RANKL antibodies $(3 \mathrm{mg} / \mathrm{kg})$, zoledronate $(0.2 \mathrm{mg} / \mathrm{kg})$, or saline (control) was injected into the vertex of the head. After $5 \mathrm{~d}$, calvarial portions were excised and fixed in $70 \%$ ethanol for $1 \mathrm{~h}$. Subsequently, the specimens were examined using micro-computed tomography $(\mu \mathrm{CT})$ to analyze calvarial structures as well as histomorphological analysis to detect osteoclasts. ${ }^{9)}$ The most effective dosages of LPS, anti-RANKL antibodies, and zoledronate administered to the mice were selected on the basis of previously reported findings. ${ }^{10-12)}$

Detection of Osteoclasts in Calvaria Calvarial specimens excised from mice were fixed in $70 \%$ ethanol for tartrate-resistant acid phosphatase (TRAP; a marker of osteoclasts) staining using a conventional method with Naphtol AS-MX phosphate (Sigma-Aldrich) and Fast red (SigmaAldrich) dissolved in $0.1 \mathrm{M}$ acetic buffer ( $\mathrm{pH}$ 5.0) containing $1 \%$ tartrate acid (Wako Pure Chemical Industries, Ltd., Osaka,
A
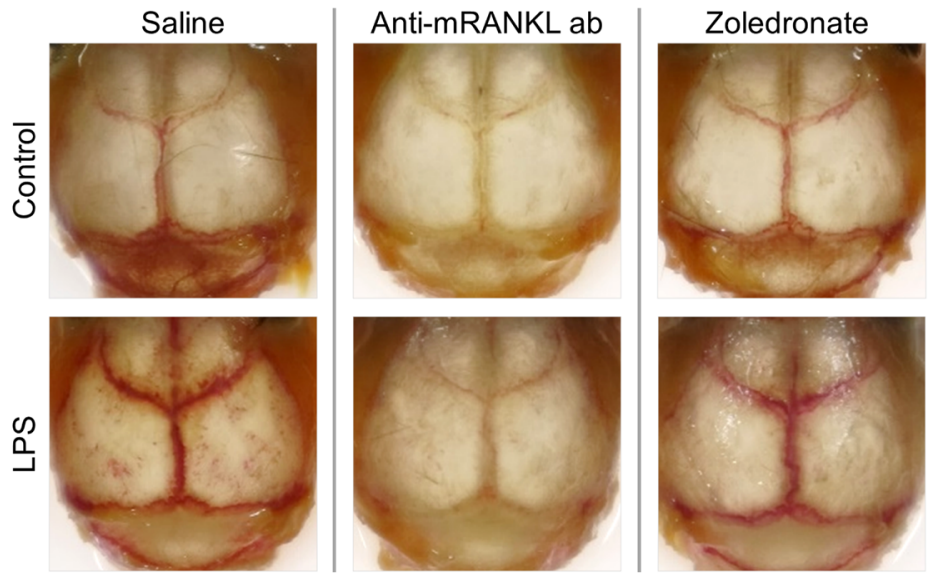

B
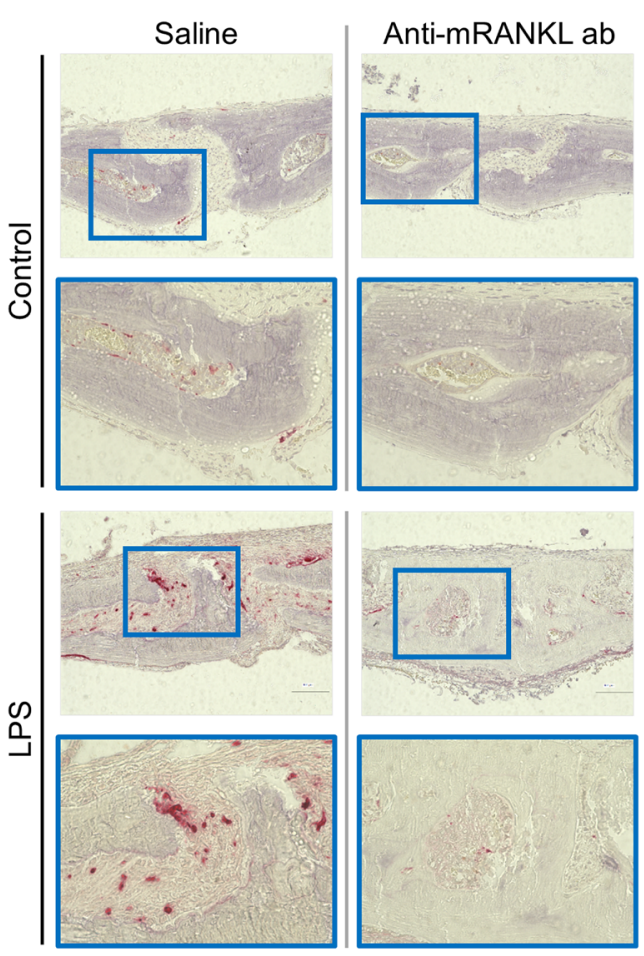

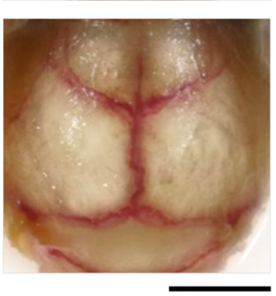

Zoledronate
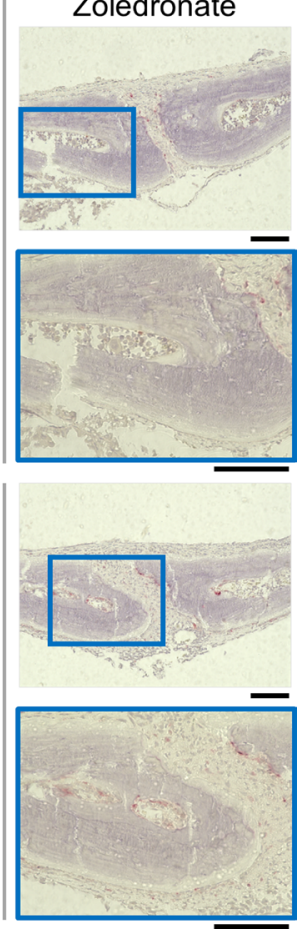

Fig. 1. Effects of Anti-mouse RANKL Antibodies and Zoledronate on LPS-Induced Calvarial Bone Destruction

Saline, anti-mouse RANKL antibodies $(3 \mathrm{mg} / \mathrm{kg})$, or zoledronate $(0.2 \mathrm{mg} / \mathrm{kg})$ with or without LPS $(25 \mathrm{mg} / \mathrm{kg})$ were each suspended in type I collagen gel, then injected into the calvaria area. After $5 \mathrm{~d}$, calvaria specimens were excised and stained for TRAP (A). Excised specimens were also cut and the coronal plane stained for TRAP (B). Areas stained in red indicated the existence of osteoclasts. Representative photographs are shown. Bar in $\mathrm{A}=5 \mathrm{~mm}$. Bars in $\mathrm{B}=0.1 \mathrm{~mm}$. 
Japan). ${ }^{13)}$ Fixed specimens were embedded in O.C.T. compound (Sakura Finetek Japan Co., Ltd., Tokyo, Japan) and cut into coronal sections, which were stained for TRAP. ${ }^{13)}$

Experimental Periodontitis Model Under anesthesia with isoflurane inhalation, the cervix of the maxillary left second molar in mice was ligated with a silk suture (size 5-0, MATSUDAIKA KOGYO Co., Ltd., Tokyo, Japan), ${ }^{14)}$ then an intraperitoneal administration of saline $(200 \mu \mathrm{L})$, anti-RANKL antibodies $(3 \mathrm{mg} / \mathrm{kg})$, or zoledronate $(0.2 \mathrm{mg} / \mathrm{kg})$ was given. At 0,1 , and 2 weeks after ligation and drug administration, each mouse head was subjected to $\mu \mathrm{CT}$ scanning under anesthesia. The ligatures in all mice remained in place throughout the experimental period. The exposed length of the tooth root was calculated by comparing $\mu \mathrm{CT}$ images obtained at 0 and 2 weeks.

$\boldsymbol{\mu C T}$ Analysis Three-dimensional digital images of the calvaria in LPS-induced bone destruction model mice were reconstructed from $\mu \mathrm{CT}$ analysis findings using a ScanXmateL090H (Comscantecno, Co., Ltd., Yokohama, Japan). Histo- morphological analysis of the $\mu \mathrm{CT}$ images was performed using a TRI/3D-Bon ${ }^{\circledR}$ system (RATOC System Engineering Co., Ltd., Tokyo, Japan). Three-dimensional digital images of alveolar bones and teeth in periodontitis model mice were reconstructed from $\mu \mathrm{CT}$ analysis findings using an in vivo $3 \mathrm{D}$ $\mu \mathrm{CT}$ system (R_mCT2, Rigaku Co., Ltd., Tokyo, Japan).

Statistical Analysis Statistical analyses were performed using the IBM PASW ${ }^{\circledR}$ statistical software package, version 18.0 (IBM, Chicago IL, U.S.A.). Values are presented as the mean \pm S.D. (standard deviation). A $p$ value $<0.05$ was considered to be significant. For experiments involving 6 factors, data were analyzed using one-way ANOVA or Student's $t$-test.

\section{RESULTS}

Administration of Anti-mouse RANKL Antibodies but Not Zoledronate Inhibits Osteoclast Formation Induced by LPS in Calvaria Denosumab does not bind to mouse RANKL, thus we used anti-mouse RANKL antibodies in
A

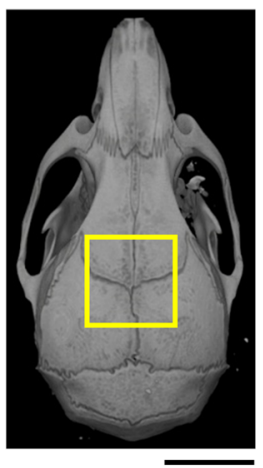

$\mathrm{B}$
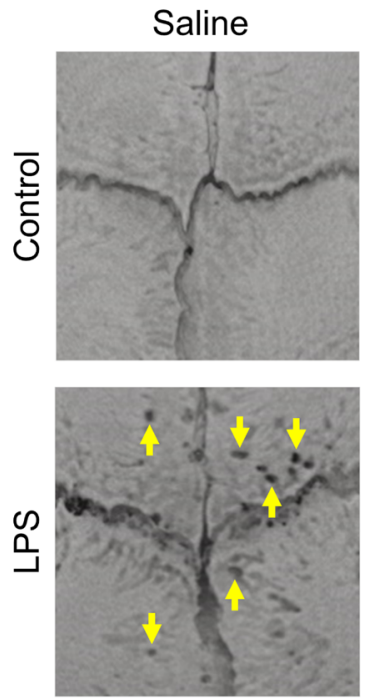

C

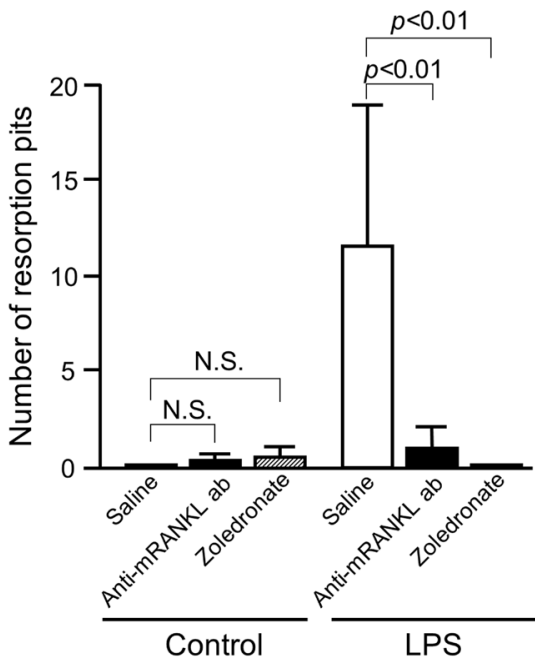

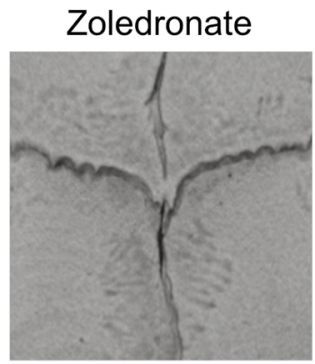
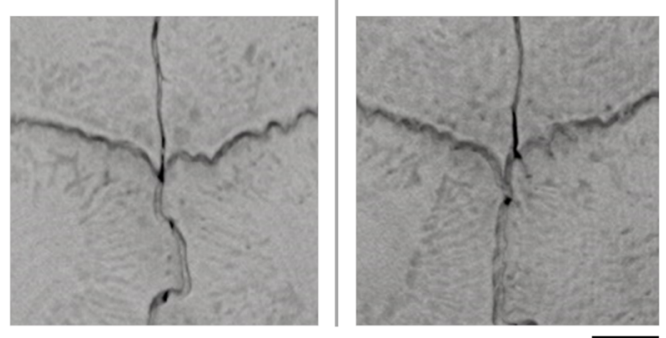

Fig. 2. Effects of Anti-mouse RANKL Antibodies and Zoledronate on Resorption Pit Formation in Calvaria Induced by LPS

Saline, anti-mouse RANKL antibodies $(3 \mathrm{mg} / \mathrm{kg})$, or zoledronate $(0.2 \mathrm{mg} / \mathrm{kg})$ with or without LPS $(25 \mathrm{mg} / \mathrm{kg})$ were each suspended in type I collagen gel and injected into the calvaria area. After $5 \mathrm{~d}$, calvaria specimens were excised and analyzed by $\mu \mathrm{CT}$ to construct 3 -D images (A, B). The numbers of resorption pits in areas indicated with a yellow frame were counted (A-C). Arrows indicate resorption pits (B). Bar in $\mathrm{A}=5 \mathrm{~mm}$. Bar in $\mathrm{B}=1 \mathrm{~mm}$. Data are shown as the mean \pm S.D. ( $n=6-8$ per group). N.S., not significant. 
this study. Initially, we examined the effects of anti-mouse RANKL antibodies on LPS-induced osteoclast formation in calvarial specimens. Following injection of LPS with saline, an increase in size of TRAP-positive areas, indicating osteoclast formation, was found along calvaria sutures as compared to the control mice (Figs. 1A, 1B, left upper and lower panels). In contrast, simultaneous injection with anti-mouse RANKL antibodies resulted in dramatically smaller TRAP-positive areas induced by LPS (Figs. 1A, 1B, lower middle panels). In addition, administration of anti-mouse RANKL antibodies reduced the size of the primary TRAP-positive areas in the control mice (Figs. 1A, 1B, upper middle panels). On the other hand, zoledronate failed to inhibit the increase in size of TRAP-positive areas in the LPS-injected and control mice (Figs. 1A, 1B, right upper and lower panels). These results indicate that anti-mouse RANKL antibodies inhibit osteoclast formation induced by LPS in the calvaria, while zoledronate does not have such an effect.

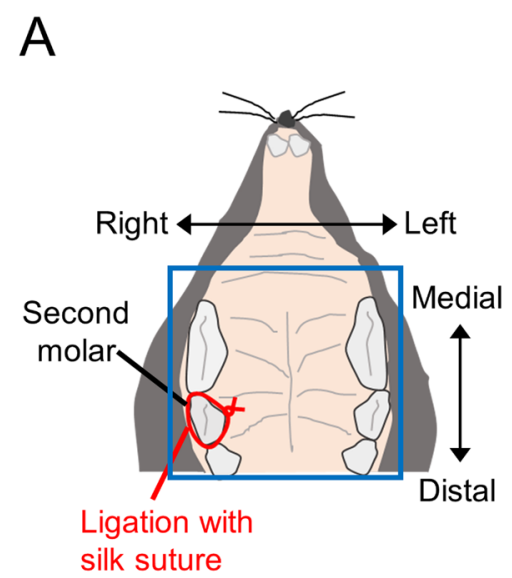

Anti-mouse RANKL Antibodies and Zoledronate Inhibit Bone Resorption Induced by LPS in Calvaria Next, we examined the effects of anti-mouse RANKL antibodies and zoledronate on calvarial bone destruction induced by LPS (Figs. 2A, B, C). The square in Fig. 2A encloses the portion of the calvaria where the number of resorption pits formed by osteoclasts was counted. The number of resorption pits in LPSinjected mice was significantly increased (Figs. 2B left lower panel, 2C) as compared to the control mice, while injection of anti-mouse RANKL antibodies or zoledronate with LPS resulted in markedly inhibited resorption pit formation (Figs. 2B middle and right lower panels, $2 \mathrm{C}$ ). These results indicate that both anti-mouse RANKL antibodies and zoledronate inhibit calvarial bone destruction induced by LPS.

Anti-mouse RANKL Antibodies Inhibit Alveolar Bone Destruction More Effectively than Zoledronate in Periodontitis Model Mice To examine the effects of anti-mouse RANKL antibodies and zoledronate on alveolar bone destruc-

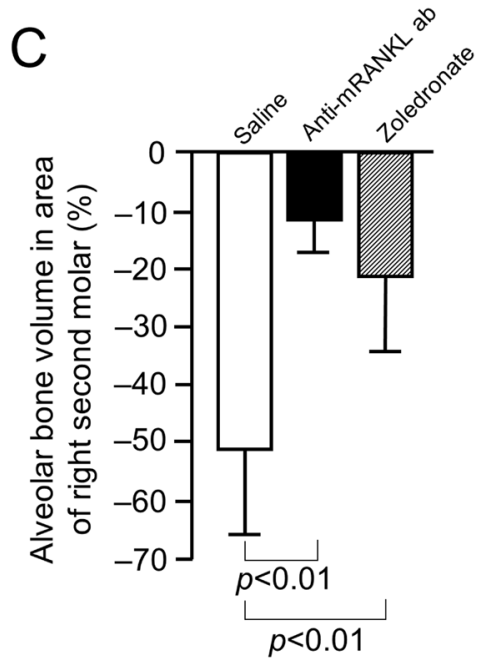

B

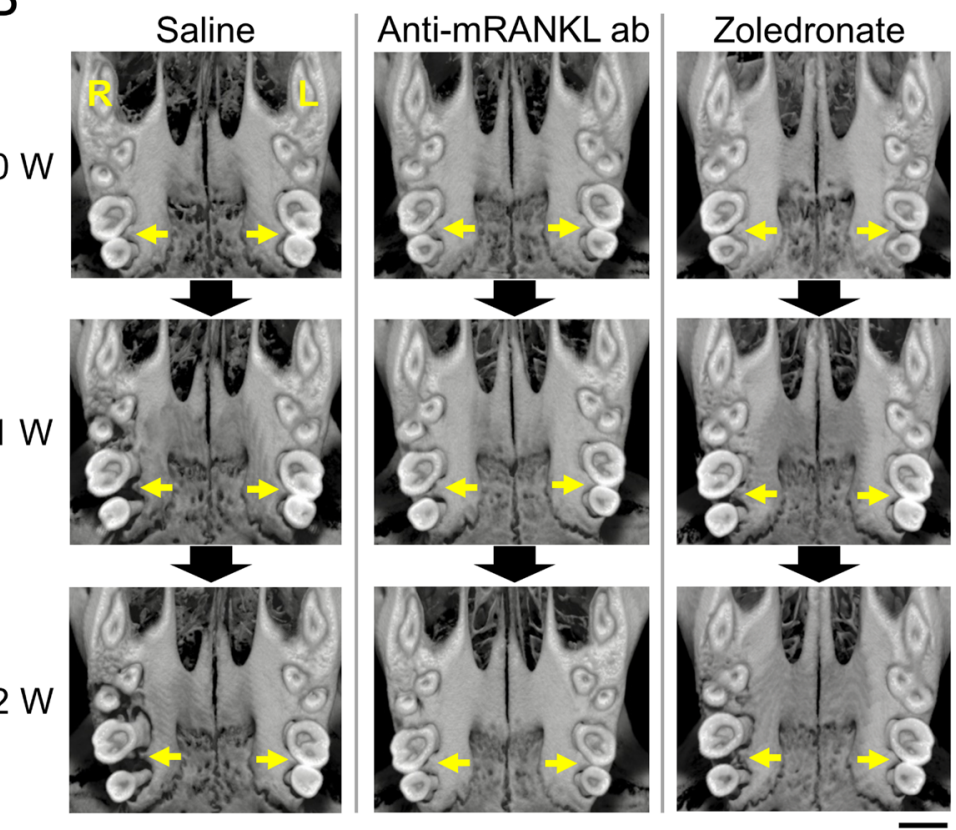

Fig. 3. Effects of Anti-mouse RANKL Antibodies and Zoledronate on Alveolar Bone Destruction in Periodontitis Model Mice

(A) Schema for producing periodontitis model mice, in which the right second molars were ligated with a silk suture. After 0 , 1, and 2 weeks, oral cavities were scanned and analyzed by $\mu \mathrm{CT}$ to construct 3-D images (B). Blue frame in (A) indicates area of oral cavity presented in (B). Alveolar bone volume in the area of the right second molars was analyzed by $\mu \mathrm{CT}(\mathrm{C})$. Data are expressed as the mean \pm S.D. $(n=6)$. Bar $=1 \mathrm{~mm}$. 
tion caused by periodontitis, we performed intraperitoneal administrations of those agents in mice following ligation of the upper-right second molar with a silk suture to induce periodontal inflammation (Figs. 3A, B, C). Figure 3B shows representative $\mu \mathrm{CT}$ images of upper jaws of periodontitis model mice at 0,1 , and 2 weeks after silk suture ligation of the right molars. After 1 and 2 weeks, apparent loss of alveolar bone was observed around the right but not the left molars in the control mice, which had received an injection of saline (Figs. $3 \mathrm{~B}$, left lane panels, 3C). On the other hand, alveolar bone loss in mice that received an injection of anti-mouse RANKL antibodies was significantly inhibited (Figs. 3B, middle lane panels, 3C). In the zoledronate-injected mice, partial inhibition of alveolar bone loss was observed (Figs. 3B, right lane panels, 3C).

Figure 4 shows representative $\mu \mathrm{CT}$ images of buccal side right molars and alveolar bone samples from periodontitismodel mice. In the control mice that received an injection of saline only, molar root exposure due to alveolar bone destruction occurred within 1 week after silk suture ligation of the right molar (Figs. 4A left lane panels, 4B, 4C). Two weeks later, alveolar bone destruction had stopped, though molar root exposure remained (Figs. 4A left lane panels, 4B, 4C). On the other hand, administration of anti-mouse RANKL antibodies significantly inhibited alveolar bone destruction and exposure of the molar roots as compared to the controls (Figs. 4A middle lane panels, 4B, 4C). The inhibitory effects of zoledronate on alveolar bone destruction was not significantly different as compared to the control, though a tendency for suppression of alveolar bone destruction was observed (Figs. 4A right lane panels, 4B, 4C). Together, these results suggest that anti-mouse RANKL antibodies protect against destruction of alveolar bone associated with periodontitis in a more effective manner than zoledronate.

\section{DISCUSSION}

Application of minocycline, an antimicrobial drug, to periodontal pockets is generally performed to treat periodontitis. ${ }^{15)}$ However, such antimicrobial agents do not show inhibitory

A

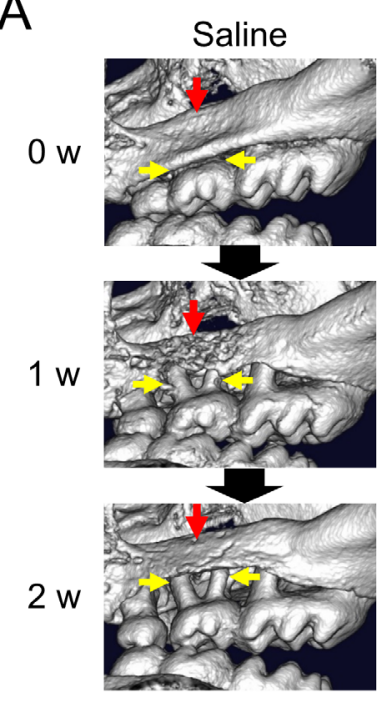

B

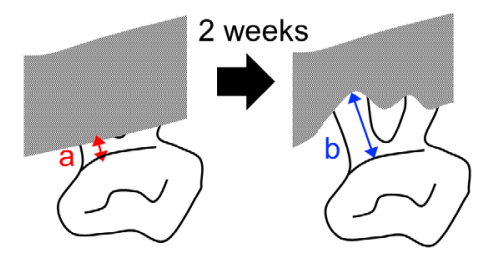

Length $(\mu \mathrm{m})$ of exposed tooth root

$$
=\mathrm{b}-\mathrm{a}
$$
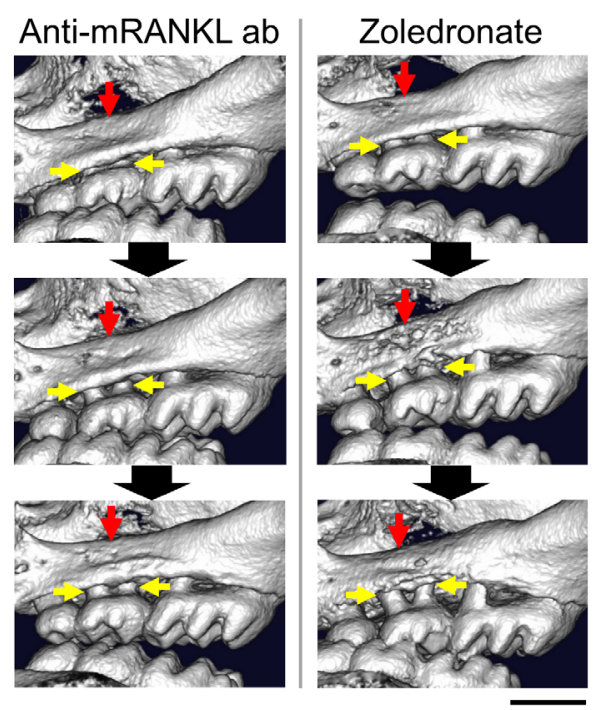

C

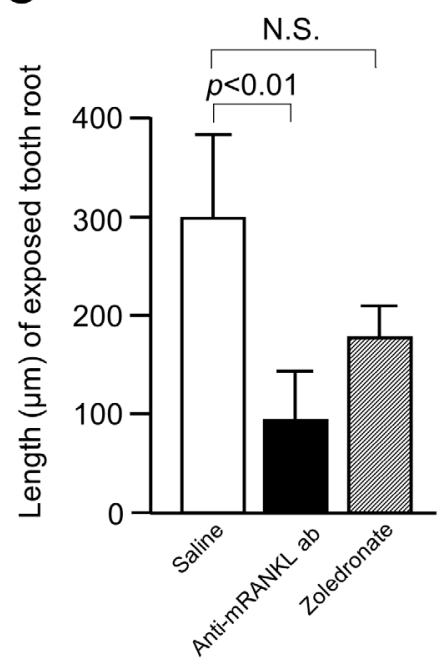

Fig. 4. Effects of Anti-mouse RANKL Antibodies and Zoledronate on Tooth Root Exposure in Periodontitis Model Mice

The right second molars in periodontitis model mice were ligated with a silk suture. After $0(0 \mathrm{w}), 1(1 \mathrm{w})$, and $2(2 \mathrm{w})$ weeks, the oral cavities were scanned and analyzed by $\mu \mathrm{CT}$ to construct 3-D images (A). Yellow arrows indicate tooth roots and red arrows alveolar bone. (B) Formula used to calculate length of tooth roots exposed after 2 weeks. (C) Length of exposed tooth roots at 2 weeks after ligation of second molars. Data are shown as the mean \pm S.D. N.S., not significant. Bar $=1$ mm. 
effects toward alveolar bone destruction in patients with severe periodontitis. Thus, novel drugs to prevent alveolar bone destruction related to periodontitis are anticipated. In the present study, we found that administration of anti-RANKL antibodies strongly suppressed calvarial bone destruction induced by LPS and alveolar bone destruction associated with periodontitis in model mice. Our results suggest that denosumab would be applicable for prevention of alveolar bone destruction seen in periodontitis patients.

LPS, a major constituent of bacteria, induces inflammatory cytokines and prostaglandins via Toll-like receptors, and those inflammatory factors have been suggested to induce alveolar bone destruction associated with periodontitis by enhancing osteoclast differentiation. ${ }^{16,17)}$ In the present LPS-induced calvarial bone destruction model, local administration of antimouse RANKL antibodies into the calvaria region reduced the number of osteoclasts and suppressed resorption pit formation, indicating that even though production of inflammatory factors was induced by LPS, RANKL signaling is essential to induce osteoclast formation. In contrast, zoledronate failed to reduce the number of osteoclasts induced by LPS, suggesting that its action is independent of RANKL signaling. Weinstein et $a l{ }^{18)}$ reported that the number of giant osteoclasts without bone resorption activity was increased in osteoporosis patients who had been administrated alendronate for a long period and those findings support our results showing that administration of anti-RANKL antibodies, but not zoledronate, can inhibit osteoclast differentiation induced by LPS.

In the present experimental periodontitis model mice, severe alveolar bone destruction was found around the molars within 1 week after ligation with a silk suture, which resulted in tooth root exposure. Although administration of antiRANKL antibodies as well as zoledronate inhibited alveolar bone destruction, the inhibitory effect shown by zoledronate was lower. Similarly, anti-RANKL antibodies but not zoledronate significantly inhibited tooth root exposure. Therefore, we consider that denosumab may be more useful for prevention of alveolar destruction associated with periodontitis than zoledronate.

The reason why zoledronate did not inhibit alveolar bone as well as the anti-mouse RANKL antibodies is not clear. We speculate that the microenvironmental condition of alveolar bone destruction in periodontitis model mice was somehow different as compared to that of osteoporosis, thus resulting in our findings for the action of zoledronate. Systematic administration with intraperitoneal injection of zoledronate or anti-RANKL antibodies significantly increased femur bone mass in the periodontitis model, whereas neither given as a local injection showed such an effect (Supplementary Fig. 1). Pharmacokinetic monitoring of alveolar bones and femurs affected by periodontitis for comparisons of zoledronate and anti-RANKL antibodies is necessary to reveal the different action mechanisms of these drugs.

Anti-resorptive agent-related osteonecrosis of the jaw (ARONJ) is induced by bisphosphonates and denosumab, and an important clinical problem. ${ }^{19)}$ In our experiments, osteonecrosis was not observed in the LPS-induced calvarial bone destruction or experimental periodontitis model (data not shown). However, the possibility of ARONJ development induced by such drugs in periodontitis patients cannot be denied. Additional experiments with long-term administration of
anti-RANKL antibodies in mice are necessary prior to clinical application. In conclusion, our results suggest that denosumab is a promising drug for prevention of alveolar bone destruction associated with periodontitis.

The present study shows results of pre-treatment with anti-RANKL antibodies in periodontitis model mice, whereas post-treatment effects were not examined. We speculate that anti-RANKL antibodies interrupt destruction and stop the decrease in alveolar bone volume. On the other hand, a dramatic increase in alveolar bone volume is not anticipated when the regenerative potency of alveolar bone is reduced in patients. Additional analyses are necessary to reveal the precise effects of denosumab on periodontal bone disease.

Acknowledgments We thank Dr. Haruka Fukamachi, Dr. Hirobumi Morisaki, and Professor Hirotaka Kuwata (Department of Microbiology, School of Dentistry, Showa University) for their technical assistance. This work was supported in part by the Private University Research Branding Project of the Ministry of Education, Culture, Sports, Science and Technology (MEXT) of Japan to Showa University, and the Industry to Support Private Universities Building up Their Foundations of Strategic Research of MEXT (S1411009, S1201014, S0801016) to M.T., Grant-in-Aids for Scientific Research (B) to M. T. (Nos. 24659830, 26293398) and to T. N.-K. (No. 25293066), and Grant-in-Aids for Scientific Research (C) to N. S. (No. 17K11993), Grant-in-Aid for Challenging Exploratory Research to T. N.-K. (No. 15K15538), Grant-in-Aid for Young Scientists (B) to A. K. (No. 16K20655), Grant-in-Aid for Scientific Research on Innovative Areas to M. C. (No. 16H01635), and Grant-in-Aid for Challenging Exploratory Research to M. C. (No. 16K15778) from the Japan Society for the Promotion of Science, as well as Grants-in-Aid from The Science Research Promotion Fund to A. K., The Uehara Memorial Foundation to T. N.-K, The Naito Foundation to T. N.-K, the Takeda Science Foundation to T. N.-K, and the Moritani Scholarship Foundation to M. C.

Conflict of Interest The authors declare no conflict of interest.

Supplementary Materials The online version of this article contains supplementary materials.

\section{REFERENCES}

1) Seeman E, Delmas PD. Bone quality - the material and structural basis of bone strength and fragility. N. Engl. J. Med., 354, 22502261 (2006)

2) Kostenuik PJ, Nguyen HQ, McCabe J, Warmington KS, Kurahara C, Sun N, Chen C, Li L, Cattley RC, Van G, Scully S, Elliott R, Grisanti M, Morony S, Hong LT, Asuncion F, Li X, Ominsky MS, Stolina M, Dwyer D, Dougall WC, Hawkins N, Boyle WJ, Simonet WS, Sullivan JK. Denosumab, a fully human monoclonal antibody to RANKL, inhibits bone resorption and increases BMD in knockin mice that express chimeric (murine/human) RANKL. J. Bone Miner. Res., 24, 182-195 (2009).

3) Kong YY, Feige U, Sarosi I, Bolon B, Tafuri A, Morony S, Capparelli C, Li J, Elliott R, McCabe S, Wong T, Campagnuolo G, Moran E, Bogoch ER, Van G, Nguyen LT, Ohashi PS, Lacey DL, Fish E, Boyle WJ, Penninger JM. Activated T cells regulate bone loss and joint destruction in adjuvant arthritis through osteoprotegerin 
ligand. Nature, 402 (6763suppl.), $43-47$ (1999).

4) Suda T, Takahashi N, Udagawa N, Jimi E, Gillespie MT, Martin TJ. Modulation of osteoclast differentiation and function by the new members of the tumor necrosis factor receptor and ligand families. Endocr. Rev., 20, 345-357 (1999).

5) Hanada R, Hanada T, Sigl V, Schramek D, Penninger JM. RANKL/ RANK-beyond bones. J. Mol. Med., 89, 647-656 (2011).

6) Bartold PM, Cantley MDHD, Haynes DR. Mechanisms and control of pathologic bone loss in periodontitis. Periodontology 2000, 53, 55-69 (2010).

7) Tanabe N, Maeno M, Suzuki N, Fujisaki K, Tanaka H, Ogiso B, Ito $\mathrm{K}$. IL-1 $\alpha$ stimulates the formation of osteoclast-like cells by increasing $\mathrm{M}-\mathrm{CSF}$ and $\mathrm{PGE}_{2}$ production and decreasing OPG production by osteoblasts. Life Sci., 77, 615-626 (2005).

8) Kressin NR, Boehmer U, Nunn ME, Spiro A 3rd. Increased preventive practices lead to greater tooth retention. J. Dent. Res., $\mathbf{8 2}$, 223-227 (2003).

9) Okamatsu N, Sakai N, Karakawa A, Kouyama N, Sato Y, Inagaki K, Kiuchi Y, Oguchi K, Negishi-Koga T, Takami M. Biological effects of anti-RANKL antibody administration in pregnant mice and their newborns. Biochem. Biophys. Res. Commun., 491, 614621 (2017).

10) Zhao B, Takami M, Yamada A, Wang $X$, Koga $T, H u X$, Tamura $T$, Ozato K, Choi Y, Ivashkiv LB, Takayanagi H, Kamijo R. Interferon regulatory factor-8 regulates bone metabolism by suppressing osteoclastogenesis. Nat. Med., 15, 1066-1071 (2009).

11) Furuya $Y$, Mori $K$, Ninomiya $T$, Tomimori $Y$, Tanaka $S$, Takahashi $\mathrm{N}$, Udagawa $\mathrm{N}$, Uchida $\mathrm{K}$, Yasuda $\mathrm{H}$. Increased bone mass in mice after single injection of anti-receptor activator of nuclear factor- $\kappa \mathrm{B}$ ligand-neutralizing antibody: evidence for bone anabolic effect of parathyroid hormone in mice with few osteoclasts. J. Biol. Chem., 286, 37023-37031 (2011)

12) Abe T, Sato T, Kokabu S, Hori N, Shimamura Y, Sato T, Yoda T.
Zoledronic acid increases the circulating soluble RANKL level in mice, with a further increase in lymphocyte-derived soluble RANKL in zoledronic acid- and glucocorticoid-treated mice stimulated with bacterial lipopolysaccharide. Cytokine, 83, 1-7 (2016).

13) Mochizuki A, Takami M, Kawawa T, Suzumoto R, Sasaki T, Shiba A, Tsukasaki H, Zhao B, Yasuhara R, Suzawa T, Miyamoto Y, Choi Y, Kamijo R. Identification and characterization of the precursors committed to osteoclasts induced by TNF-related activationinduced cytokine/receptor activator of NF- $k \mathrm{~B}$ ligand. J. Immunol., 177, 4360-4368 (2006)

14) Abe T, Hajishengallis G. Optimization of the ligature-induced periodontitis model in mice. J. Immunol. Methods, 394, 49-54 (2013).

15) Paquette DW, Hanlon A, Lessem J, Williams RC. Clinical relevance of adjunctive minocycline microspheres in patients with chronic periodontitis : secondary analysis of a phase 3 trial. J. Periodontol., 75, 531-536 (2004).

16) Myokai F, Takashiba S, Lebo R, Amar S. A novel lipopolysaccharide-induced transcription factor regulating tumor necrosis factor alpha gene expression: molecular cloning, sequencing, characterization, and chromosomal assignment. Proc. Natl. Acad. Sci. U.S.A., 96, 4518-4523 (1999).

17) Suda K, Udagawa N, Sato N, Takami M, Itoh K, Woo J, Takahashi $\mathrm{N}$, Nagai K. Suppression of osteoprotegerin expression by prostaglandin $\mathrm{E}_{2}$ is crucially involved in lipopolysaccharide-induced osteoclast formation. J. Immunol., 172, 2504-2510 (2004).

18) Weinstein RS, Roberson PK, Manolagas SC. Giant osteoclast formation and long-term oral bisphosphonate therapy. N. Engl. J. Med., 360, 53-62 (2009).

19) Aghaloo T L, Cheong S, Bezouglaia O, Kostenuik P, Atti E, Dry SM, Flavia Q, Tetradis S. RANKL inhibitors induce osteonecrosis of the jaw in mice with periapical disease. J. Bone Miner. Res., 29, $843-854$ (2014). 